\title{
Efecto Residual de la Fertilización del Cultivo de Papa Sobre el Cultivo de Haba (Vicia faba L) en el Sistema de Rotación
}

\author{
Bruno Condori ${ }^{1}$, André Devaux ${ }^{2}$ Pablo Mamani, ${ }^{1}$ Juan \\ Vallejos, ${ }^{1}$ Jorge Blajos ${ }^{1}$
}

\begin{abstract}
Resumen
Durante la campaña agrícola 1993-1994, en la Estación Experimental Toralapa en Cochabamba, Bolivia, se evaluó el efecto residual de niveles de fertilización mineral (nitrógeno y fósforo) y de la aplicación de cal viva $\left(\mathrm{Ca}(\mathrm{OH})_{2}\right)$ en el comportamiento agronómico del haba (Vicia faba L.). Este estudio es la continuación de trabajos iniciados por el Programa de Investigación de la Papa (PROINPA), sobre la fertilización del cultivo de la papa en 1991-1992 y los efectos residuales en los cultivos de rotación como la cebada y el haba. Los análisis de suelos mostraron que el nutriente más afectado por los niveles de fertilización fue el fósforo, con una alta disponibilidad en el suelo en el segundo año de rotación. En cambio, el efecto residual de la aplicación de cal incrementó los valores de $\mathrm{pH}$, calcio, magnesio y la Capacidad de Intercambio Catiónico (CIC). Los principales resultados indican que el efecto residual de la cal, independientemente de las dosis aplicadas (10 ó 20t/ha), permiten incrementar los componentes del rendimiento en materia seca de haba: la fitomasa aérea de $49 \%$, la nodulación bacteriana (223\%), y el rendimiento en grano seco (46\%) con respecto al testigo. Esto demuestra el prolongado efecto residual de la cal y su efecto positivo en la productividad del cultivo de haba. Se determinó también que el cultivo de haba es el segundo cultivo más rentable después de la papa en el sistema de rotación que también incluye la cebada. El valor actualizado neto (VAN) para el sistema de rotación (papa, cebada y haba) muestra
\end{abstract}

Aceptado para publicación: julio 1997.

\footnotetext{
${ }^{1}$ Investigadores, Programa de Investigación de la Papa - PROINPA (Convenio IBTA-CIP-COSUDE), Casilla 4285, Cochabamba, Bolivia.

${ }^{2}$ Investigador CIP y Co-Director, PROINPA.
} 
que el nivel de 40-80-0 kg/ha de $\mathrm{N}_{\text {y }} \mathrm{P}_{2} \mathrm{O}_{5}$ combinado con 10 tha de cal viva es el que proporciona el mayor beneficio con $12,520 \mathrm{Bs} / \mathrm{ha}$. (US\$1=Bs 4.65). Se necesita continuar los estudios para establecer dosis óptimas de cal.

Palabras claves adicionales: efecto residual, papa, fertilización, cal, haba, nitrofijación, análisis económico.

\title{
Residual Effect of the Fertilization of the Potato Crop on the Faba Bean Crop (Vicia faba L.) in the Rotation System
}

\begin{abstract}
Summary
During 1993-1994 at the Toralapa Experimental Research Station in Cochabamba, Bolivia, the residual effect of mineral fertilization levels (nitrogen and phosphorus) and of calcium hydroxide $\left(\mathrm{Ca}(\mathrm{OH})_{2}\right)$ application, was evaluated on the production of the faba bean crop (Vicia faba L.). This study continues activities initiated in 1991-1992 by the Bolivian National Potato Research Program (PROINPA), on the fertilization of the potato crop, and its residual effect on the rotation crops such as barley and faba bean.

The soil analysis showed that phosporus was the nutrient most affected by the fertilization levéis, with a high availability in the soil in the second year of rotation. On the other hand, the residual effect of $\mathrm{Ca}(\mathrm{OH})_{2}$ application increased the valúes of $\mathrm{pH}$, calcium, magnesium and the Cationic Exchange Capacity. The main results indícate that the residual effect of $\mathrm{Ca}(\mathrm{OH})_{2}$, independently of the rates applied (10 or $20 \mathrm{t} / \mathrm{ha}$ ), increase the dry matter yield component of faba bean: the aerial plant weight (49\%), the bacterial nodulation (223\%) and the yield in dry grain (46\%). This shows the prolonged residual effect of $\mathrm{Ca}(\mathrm{OH})_{2}$ applied to the soil and its positive effect on the productivity of the faba bean crop.

Economic analysis shows that faba bean is the second most profitable crop after the potato, in the rotation system which also includes barley. The present net valué for the rotation system (potato, barley, bean) shows that the level of $40-80-0 \mathrm{~kg} / \mathrm{ha}$ of $\mathrm{N}$ and $\mathrm{P}_{2} \mathrm{O}_{5}$ combined with 10 tha of $\mathrm{Ca}(\mathrm{OH})_{2}$, is the level that gives the highest benefit, obtaining 12,520 Bs/ha (US\$1 $=\mathrm{Bs} 4.65)$. Further studies are required to establish optimum $\mathrm{Ca}(\mathrm{OH})_{2}$ rates.
\end{abstract}


Additional index words: residual effect, potato, fertilization, lime, faba bean, nitrogen fixation, economic analysis.

Con la finalidad de desarrollar tecnologías cada vez más acordes a los requerimientos de los agricultores que cultivan la papa, también se toman en consideración los sistemas de producción agrícola que involucran a este cultivo. Por ello, el haba, componente del ciclo de rotación practicada por los agricultores, se enmarca en este estudio.

En las alturas de la provincia Tiraque en Cochabamba, se ha observado que el pH fluctúa entre 4.3 y 5.5 , la CIC es reducida y en general, los suelos son considerados de baja fertilidad $(6,9,17)$. Esta problemática abarca una gran superficie (11,696 ha), que en la actualidad es una de las principales zonas productoras de papa en Bolivia (5).

La producción intensiva de papa como cultivo principal, requiere de la aplicación de fertilizantes y enmiendas para optimizar el uso de los suelos. Además, la práctica de la rotación de los cultivos y de descanso, permiten minimizar problemas fitosanitarios y mejorar la fertilidad natural del suelo. Estas condiciones incrementan la productividad de los cultivos del sistema de producción (4).

La incorporación de leguminosas dentro del sistema de rotación de cultivos, otorga diversas ventajas, sobre todo aquellas productoras de grano, como el haba, que es una fuente de proteínas importantes para la alimentación humana y ganadera. Además, es la leguminosa más importante por su capacidad de mantener o mejorar la fertilidad del suelo, ya que fija la mayor cantidad de nitrógeno atmosférico $(2,7)$. Sin embargo, las leguminosas son susceptibles a condiciones de acidez del suelo y requieren cantidades altas de fósforo $(8,10,16)$.

En la actualidad en Bolivia también se aprecia la importancia económica de las leguminosas, ya que existen expectativas con demandas no cubiertas en el mercado de exportación para grano de haba de zonas altas (8). 
Por los antecedentes descritos, es importante conocer el efecto de las prácticas de fertilización y enmienda aplicados al cultivo de la papa sobre el posterior cultivo de haba, considerando su importancia como uno de los cultivos del ciclo de rotación. El objetivo principal del presente estudio, realizado en coordinación con el Proyecto Fertisuelos-FAO, fue determinar el efecto residual de niveles de fertilización mineral y de dosis de cal, en la biomasa e infección de rizobio, los rendimientos biológico y agronómico y la rentabilidad del cultivo de haba dentro del sistema de rotación papa, cebada y haba.

\section{Materiales y Métodos}

La investigación se llevó a cabo en la Estación Experimental Toralapa, perteneciente a la Provincia Tiraque del Departamento de Cochabamba (Bolivia), a una altitud de $3,430 \mathrm{msnm}$ y ubicada geográficamente a los $17^{\circ}$ $31^{\prime}$ de latitud sur y los $65^{\circ} 40^{\prime}$ de longitud oeste.

Este estudio se inició con la incorporación de fertilizantes y de cal (Tabla 1) en el cultivo de papa, variedad Waych'a (ssp. andigena) en 1991-1992. Los resultados iniciales de los análisis de suelos fueron: $\mathrm{pH}$ 5.5; materia orgánica $1.7 \%$; nitrógeno total $0.11 \%$; fósforo $19 \mathrm{ppm}$ y potasio 0.34 meq en $100 \mathrm{~g}$ de suelo. Las fuentes utilizadas para la aplicación del fertilizante fueron: úrea, superfosfato triple y cal apagada $\left(\mathrm{Ca}(\mathrm{OH})_{2}\right)$, cuya aplicación se realizó al momento de la siembra del cultivo de papa.

Posteriormente se evaluó el efecto remanente de estas prácticas de fertilización en el cultivo de cebada (var. IBTA 80) en 1992-1993, y finalmente en el cultivo de haba (var. Habilla) en 1993-1994. Este sistema de rotación es normalmente utilizado por los agricultores de la zona (4).

Para el análisis estadístico, se utilizó el diseño de bloques completos al azar con arreglo factorial de $4 \times 3$, con tres repeticiones. Los factores estudiados fueron:

A Efecto residual de la aplicación de niveles de fertilización mineral. (0-0-0, 40-80-0, 80-160-0 y 160-160-0 kg/ha de $\mathrm{N}$ y $\mathrm{P}_{2} \mathrm{O}_{5}$ )

B Efecto residual de la aplicación de cal. $\left(0,10\right.$ y 20 tha de cal $\left(\mathrm{Ca}(\mathrm{OH})_{2}\right)$ 
Tabla 1. Aplicación de niveles de fertilización mineral combinados con cal al cultivo de papa (1991-1992).

\begin{tabular}{cccccc}
\hline Tratamientos & Clave & & $\begin{array}{c}\text { Fertilizante } \\
\mathrm{N}-\mathrm{P}_{2} \mathrm{O}_{5}-\mathrm{K} \\
(\mathrm{Kg} . / \mathrm{ha})\end{array}$ & $\begin{array}{c}\text { Cal } \\
\mathrm{Ca}(\mathrm{OH})_{2} \\
(\mathrm{t} / \mathrm{ha})\end{array}$ \\
\hline 1 & a1b1 & $0-$ & $0-$ & 0 & +0 \\
2 & a1b2 & $0-$ & $0-$ & 0 & +10 \\
3 & a1b3 & $0-$ & $0-$ & 0 & +20 \\
4 & a2b1 & $40-$ & $80-$ & 0 & +0 \\
5 & a2b2 & $40-$ & $80-$ & 0 & +10 \\
6 & a2b3 & $40-$ & $80-$ & 0 & +20 \\
7 & a3b1 & $80-$ & $160-$ & 0 & +0 \\
8 & a3b2 & $80-$ & $160-$ & 0 & +10 \\
9 & a3b3 & $80-$ & $160-$ & 0 & +20 \\
10 & a4b1 & $160-$ & $160-$ & 0 & +0 \\
11 & a4b2 & $160-$ & $160-$ & 0 & +10 \\
12 & a4b3 & $160-$ & $160-$ & 0 & +20 \\
\hline
\end{tabular}

Los niveles iniciales de fertilizante y de cal aplicados al cultivo de papa se presentan en la Tabla 1. El arreglo combinatorio de los factores dio lugar a doce tratamientos.

La siembra del cultivo de haba se realizó el 8 de setiembre de 1993, en forma manual, depositando dos semillas por golpe cada $0.25 \mathrm{~m}$ y a una profundidad de $0.03 \mathrm{~m}$. La cosecha se efectuó el 15 de abril de 1994. Cada unidad experimental fue de $16.50 \mathrm{~m}^{2}$, constituida por seis surcos de $5.0 \mathrm{~m}$ de largo, espaciados a $0.58 \mathrm{~m}$ entre surcos. La distancia entre plantas fue de $0.25 \mathrm{~m}$.

Entre las principales variables de respuesta del cultivo y otros análisis auxiliares, se tomaron las siguientes:

Análisis de suelos. Se tomaron muestras de suelo al inicio del estudio, antes de la siembra del cultivo de papa (1991); al inicio de la siembra de cebada (1992); al inicio y al final del cultivo de haba (1993-1994). Se analizaron seis muestras para dicho estudio, entre ellos el testigo (0-0-0), 40-80-0 y 160-160-0 con sus respectivas.

combinaciones con 0 y 10 t/ha de cal, las que fueron analizadas en los laboratorios del CIAT- Santa Cruz, Bolivia. 
Infección de nódulos de rizobio. Se evaluaron las muestras en la maduración de vainas antes de la cosecha, precediéndose al conteo de bacteroides 0 nódulos en las raíces, obteniéndose posteriormente su biomasa (13). Las muestras se tomaron en las plantas de los dos surcos centrales para evitar el efecto de bordes.

Componentes de rendimiento. Se evaluó el número de plantas por unidad experimental, número de ramas por planta y número de vainas por planta antes de la cosecha; el número de granos por vaina y el peso seco de cien granos después de la cosecha (1).

Rendimiento biológico o fitomasa seca, se midió antes de la cosecha, separando la parte aérea (follaje) y la parte subterránea de la planta (15).

Rendimiento agronómico. Se midió en dos estadios de cosecha, en vaina verde y en grano seco.

Análisis económico. Se determinó el valor actualizado neto (VAN) o beneficio neto actualizado de los tres cultivos de la rotación, considerando la sucesión de éstas como un proyecto de inversión a mediano plazo de tres años del ciclo de rotación (11 y 14). La actualización elimina las diferencias temporales y trata a todos los valores como si pertenecieran al año base (año de inversión). La inversión comprende el costo del fertilizante, de la cal y de su aplicación. Los beneficios están constituidos por los ingresos netos correspondientes a los cultivos de papa en tubérculo para el primer año, cebada en grano para el segundo y haba en grano para el tercer año.

\section{Resultados y Discusión}

\section{Efecto residual de la aplicación de fertilizantes}

El efecto residual de la aplicación de los fertilizantes minerales en la composición química del suelo, es notorio solamente para el fósforo, cuya concentración aumentó después de la aplicación del fertilizante; este efecto se notó hasta el segundo año (1992) del sistema rotacional cuando fue sembrado el cereal (16). El nivel de fósforo en el suelo bajó a niveles inferiores al inicial a partir del tercer año, antes de la siembra del haba en 1993 (Figura 1). En esta figura, también se observa que la combinación de cal (10 t/ha) a la aplicación de fertilizantes aumenta la disponibilidad de fósforo; esto es perceptible aún en suelos que no tuvieron la aplicación de fertilizantes minerales. 
Figura 1. Contenido de fósforo en los suelos debido al residuo de fertilizante y cal (10 t/ha), después de tres años de su aplicación.

En el caso del nitrógeno, el análisis de suelos no mostró cambios durante los tres años del ciclo de rotación, manteniéndose el contenido en el suelo de $0.11 \%$.

En el caso de las variables de respuesta del cultivo de haba, el número de vainas por planta fue el único componente de rendimiento que respondió al efecto residual de los fertilizantes minerales, mostrando que en los suelos con niveles elevados de fertilización fosfórica, existe un efecto residual prolongado (Figura 2). Esto es posiblemente atribuible a la liberación gradual del fósforo por el suelo, por lo que la variable de respuesta incrementa su valor en proporción directa a los niveles de fertilización $(12,16)$. El resto de los componentes de rendimiento no reportan diferencias a ningún factor en estudio. 


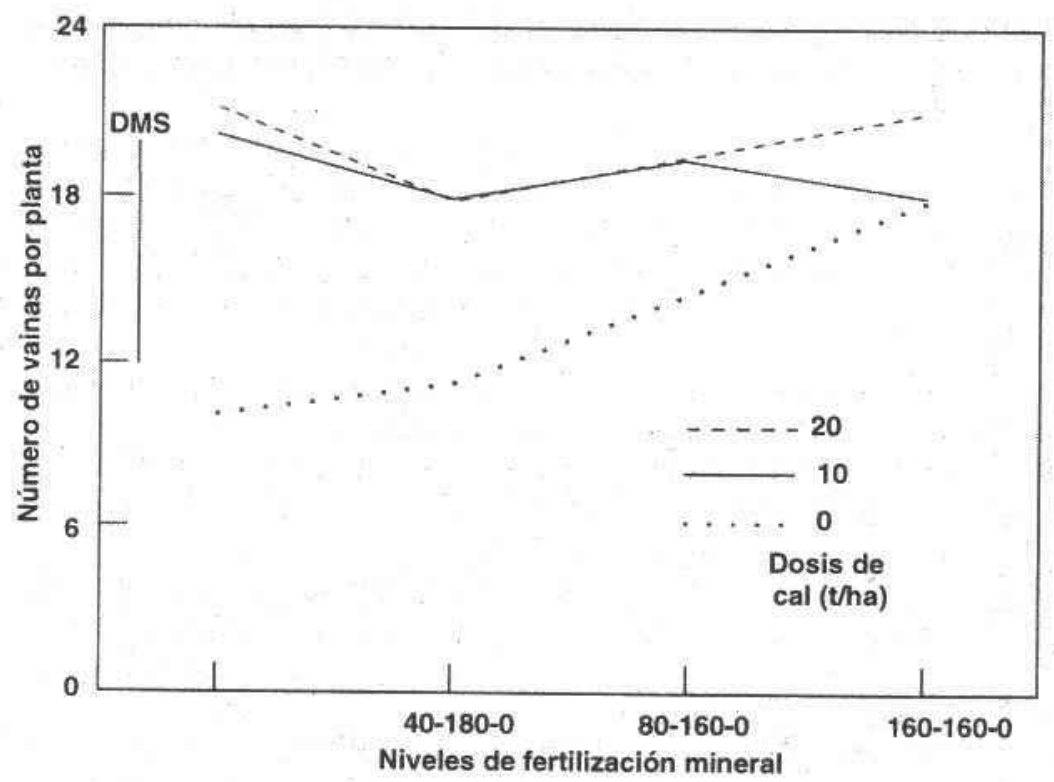

Figura 2. Interacción de niveles residuales de fertilización y cal, en el número de vainas por planta de haba (Toralapa, 1993-94).

\section{Efecto residual de la aplicación de cal}

La aplicación de cal en todos los casos (con o sin fertilizante), elevó el pH de los suelos hasta un nivel de 7, incrementando la capacidad de intercambio catiónico $(\mathrm{CIC})$, liberando calcio y magnesio intercambiables (16). Este efecto residual fue muy marcado para el segundo año (1992), pero a excepción del $\mathrm{pH}$, se redujeron los valores de calcio, magnesio y CIC a partir del tercer año, antes de la siembra del cultivo de haba (Figuras 3 y 4).

Se observó claramente que el nivel de magnesio disponible aumenta con la aplicación de cal en el segundo año de rotación, bajando su disponibilidad hasta el tercer año (Figura 4). En la misma figura también se observa que los niveles del potasio van bajando paulatinamente de niveles bajos a muy bajos. El aluminio se comporta en forma similar, ya que a partir del tercer año no se halla en el suelo en su forma disponible. Esto se podría explicar por la precipitación de aluminio debido a la aplicación de cal (17). 
Infección del rizobio. Las variables en estudio correspondientes al microsimbionte (rizobio), fueron las que arrojaron resultados más contundentes por el efecto residual de la aplicación de cal, independientemente de sus dosis (10 ó 20 t/ha). El número de nódulos por planta, se incrementa de 30 en el testigo a 136 en aquellos tratamientos con residuo de cal (10 ó 20 t/ha, indistintamente). Esta tendencia es similar en la biomasa seca de nódulos que aumentó de 185 a $598 \mathrm{mg} /$ planta (Figuras 5 y 6). Esta respuesta se debería al ambiente neutro en el suelo generado por la cal, lo que favorece el desarrollo del rizobio $(2,3,13)$.

Componentes de rendimiento: el efecto residual de la aplicación de cal influyó principalmente en el número de ramas por planta (Tabla 2).

Tabla 2. Número de ramas en el cultivo de haba, por efecto residual de fertilización y cal (1993-1994).

\begin{tabular}{cccccc}
\hline \multirow{2}{*}{$\begin{array}{c}\text { Dosis } \\
\text { de cal }\end{array}$} & \multicolumn{5}{c}{ Número de ramas por planta } \\
\cline { 2 - 5 } (t/ha) & \multicolumn{5}{c}{ Niveles de fertilizante (kg/ha) } \\
\cline { 2 - 5 } & $0-0-0$ & $40-80-0$ & $80-1604)$ & $160-160-0$ & \\
\hline 0 & 4.3 & 4.5 & 4.7 & 5.0 & $4.6 \mathrm{~B}$ \\
10 & 6.0 & 5.5 & 5.6 & 5.0 & $5.5 \mathrm{~A}$ \\
20 & 5.9 & 5.3 & 5.9 & 5.4 & $5.6 \mathrm{~A}$ \\
Promedio* & $5.4 \mathrm{~A}$ & $5.1 \mathrm{~A}$ & $5.4 \mathrm{~A}$ & $5.1 \mathrm{~A}$ & $5.2^{\star *}$ \\
\hline
\end{tabular}

\footnotetext{
* Promedios con distintas letras tanto en columnas como en filas, son diferentes estadísticamente según Duncan al 5\%.

** Promedio general
}

Rendimiento biológico. La biomasa seca de la parte aérea del haba tiene una respuesta marcada al efecto remanente de la aplicación de la cal. Por el contrario, la parte subterránea correspondiente a la raíz sin nódulos no responde a ningún factor en estudio. La fitomasa seca aérea, en relación al testigo sin cal incrementa de 96 a 143 gramos por planta (Figura 7). Esto se nota en un mejor desarrollo del cultivo, por el efecto en el pH del suelo que es neutro, disponibilidad moderada de calcio (Figura 2) y la interacción con el desarrollo del rizobio, que se integran a la fisiología general de la planta aportando con nitrógeno atmosférico para la formación de aminoácidos, fuente principal de las proteínas $(2,3,16,18)$. 


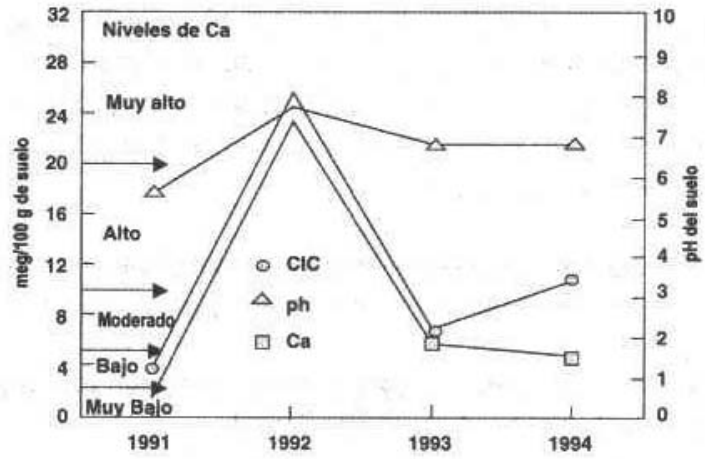

Figura 3. Contenido de calclo, valores de capacidad de intercambio catiónico y pH, luego de tres años de la aplicación de cal.

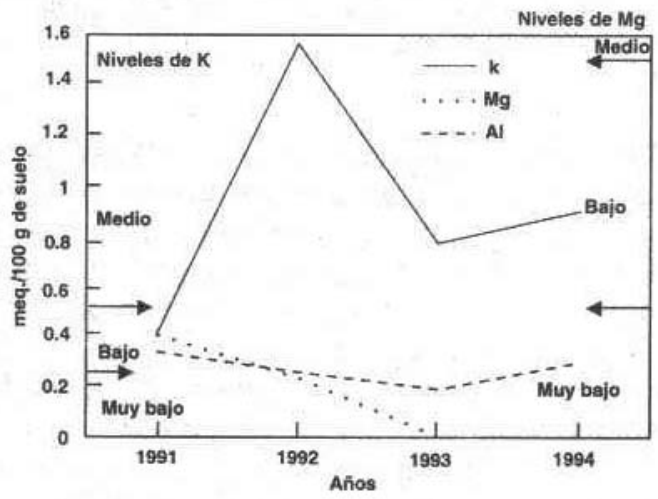

Figura 4. Contenido de potasio, magnesio y aluminio en el suelo luego de tres años de la aplicación de cal (10 t/ha). 


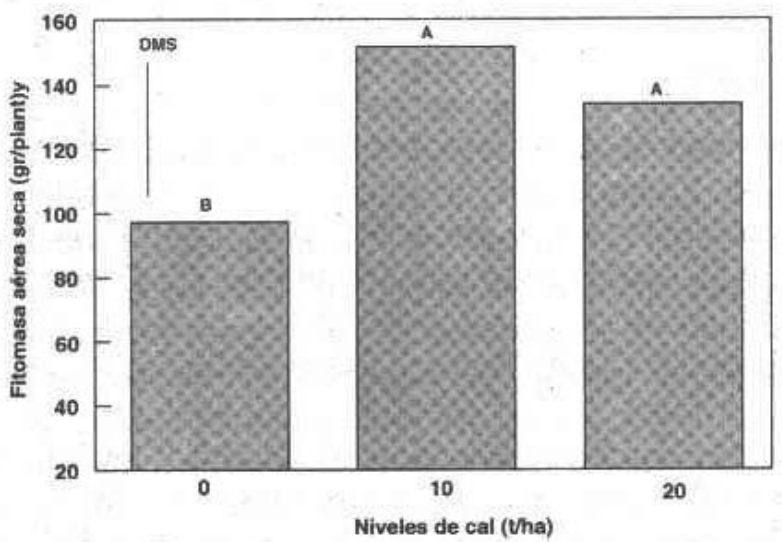

Figura 5. Efecto residual de cal en el número de nódulos en el cultivo del haba (Toralapa, 1993-94).

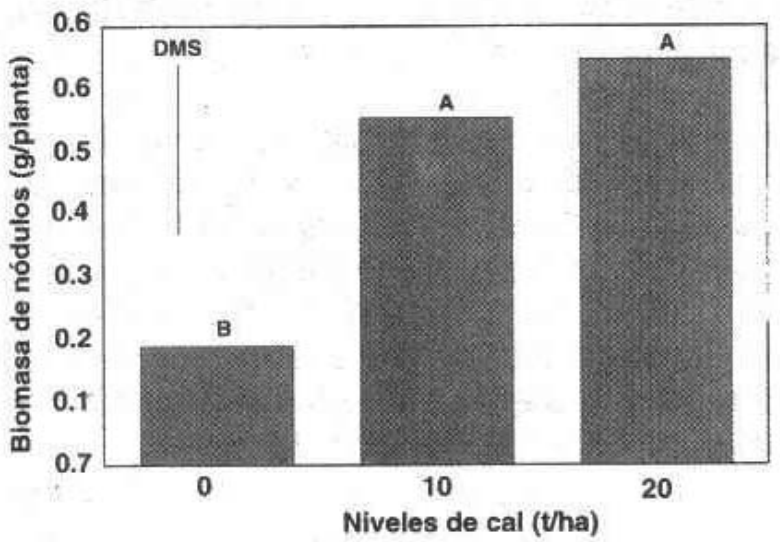

Figura 6. Efecto residual de cal en la biomasa seca de nódulos del cultivo de haba (Toralapa, 1993-94). 
Rendimiento agronómico. El rendimiento en vaina verde y en grano seco tiene una tendencia similar y asociada a la anterior variable $e$ incrementa por el efecto residual de cal, en relación al testigo, de 11.6 a 17.9 y de 2.6 a 3.9 t/ha, respectivamente (Figura 8).

\section{Análisis económico}

Considerando los costos e ingresos para todo el ciclo de rotación (papa tubérculo, cebada en grano y haba en grano), el análisis económico muestra que el valor actual neto (VAN) del nivel $40-80-0 \mathrm{~kg} / \mathrm{ha}$ de $\mathrm{N}$ y $\mathrm{P}_{2} \mathrm{O}_{5}$ combinado con $10 \mathrm{t} / \mathrm{ha}$ de cal (Tratamiento 5), es el más recomendable desde el punto de vista de la rentabilidad del ciclo de rotación con 12,520 Bs/ha (US\$2,692) (Figura 9).

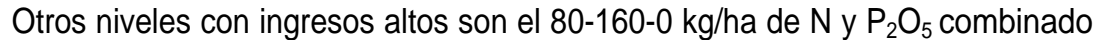
con 10 t/ha de cal (Tratamiento 8) y el $160-160-0 \mathrm{~kg} / \mathrm{ha}$ de $\mathrm{N}$ y $\mathrm{P}_{2} \mathrm{O}_{5}$ (tratamiento 10), con 12,361 y 11,814 Bs/ha, respectivamente (Figura 9). El primer nivel puede ser considerado como una alternativa de la práctica de la aplicación de fertilizantes y cal en el ciclo de rotación. El segundo nivel, se caracteriza por su beneficio neto alto y su bajo costo, que se debe principalmente a los beneficios netos obtenidos con el cultivo de papa y la respuesta marcada por este cultivo al nivel de fertilización 160-160-0 kg/ha de $\mathrm{N} \mathrm{y} \mathrm{P}_{2} \mathrm{O}_{5}$. El impacto sobre los otros cultivos de la rotación fue más reducido. Esto nos indica que tenemos que buscar la alternativa de fertilización que procure el mejor beneficio neto en el cultivo de papa con un efecto positivo en los otros cultivos de la rotación.

Considerando las tres mejores alternativas de fertilización desde el punto de vista económico (tratamientos 5,8 y 10), se determinó que la papa aporta con el $55 \%$, la cebada con el $14 \%$ y el haba con el $31 \%$ de los ingresos totales del sistema de rotación. La producción en grano seco del cultivo del haba es la que aporta con mayores beneficios económicos después del cultivo de la papa, aún sin considerar otras de sus diversas ventajas como mejoradora de las condiciones de fertilidad del suelo (Figura 10).

\section{Conclusiones}

Analizados los resultados obtenidos, se observa que la aplicación de cal tiene un efecto mucho más marcado sobre los cultivos de la rotación, como la cebada y el haba, que sobre el cultivo de papa. 
El mayor efecto residual de la aplicación de fertilizantes, constituidos de nitrógeno y fósforo, se observó principalmente con el fósforo, tanto en los análisis de suelo, como también por las respuestas de los cultivos de cebada y de haba. A pesar de que el nivel de fósforo parecía haber regresado a su nivel inicial el tercer año con el cultivo de haba, su efecto aún fue notorio en el componente de rendimiento correspondiente al número de vainas verdes por planta. Esto se debería a la disponibilidad gradual de este elemento en el suelo.

La aplicación de cal al inicio del ciclo de rotación elevó el pH del suelo incrementando la capacidad de intercambio catiónico, liberando así el calcio, fósforo y magnesio intercambiables. Este efecto fue marcado en los análisis de suelo del segundo año pero, aparte del pH, en el tercer año de rotación, el análisis de suelo mostró que los otros elementos habían regresado a su nivel inicial. Por los efectos observados en el rendimiento del haba, hay un efecto muy positivo del residuo de la aplicación de cal sobre el desarrollo y rendimiento de este cultivo. Podríamos considerar que el $\mathrm{pH}$ neutro alcanzado, favorece la liberación de cationes intercambiables y la formación de nódulos nitrificadores, lo que favoreció el desarrollo del cultivo de haba con un efecto positivo sobre la fertilidad del suelo. Este efecto continuará siendo analizado en el próximo cultivo de papa que iniciará una nueva rotación después del haba.

No se observó diferencia alguna entre los efectos residuales de las dos dosis iniciales de cal, 10 y 20 t/ha, aplicadas inicialmente al cultivo de papa. En base a esta experiencia, se están evaluando los efectos que se podrían alcanzar con niveles más bajos de cal. Considerando primero el costo de aplicación de la cal, su efecto sobre los ingresos netos del cultivo de papa y su impacto sobre el beneficio neto global del sistema de rotación, hay que establecer los niveles de aplicación de cal que permitan llegar a un beneficio neto óptimo.

Considerando que el cultivo de la papa es el cultivo más rentable de la rotación, hay que adaptar los cambios tecnológicos hacia una mejor rentabilidad de este cultivo, tratando de conseguir un efecto positivo sobre los otros cultivos de la rotación. Además, como el cultivo de papa se desarrolla mejor en condiciones de suelos ácidos, se deberá buscar una dosis de aplicación de cal que no afecte al cultivo de la papa, tanto al momento de la aplicación de cal, como cuando el cultivo de papa regresa en la misma parcela después de la conclusión del ciclo de rotación. Una alternativa a considerarse es que la cal se aplicaría al momento de sembrar los cereales y analizar sus efectos directo y residual sobre los otros cultivos de las campañas siguientes. 


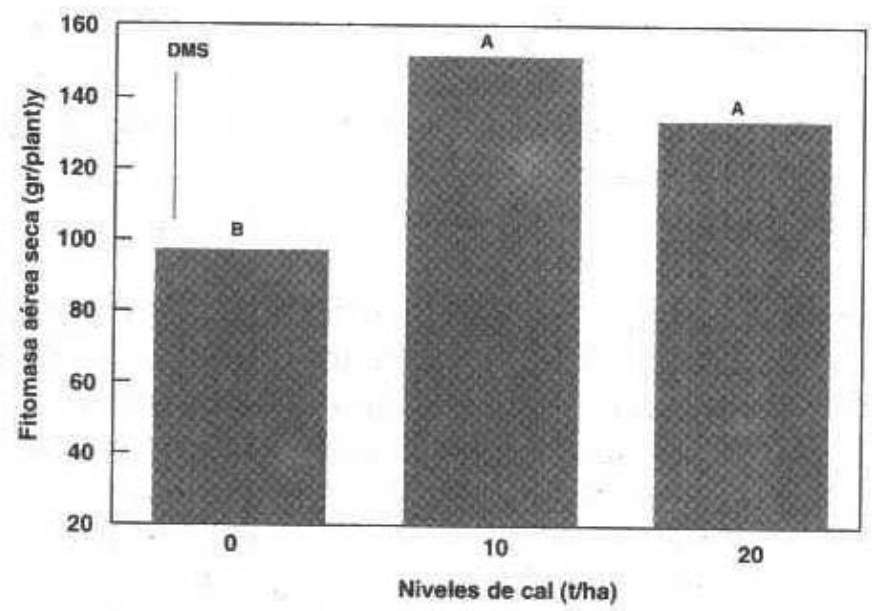

Figura 7. Efecto residual de la cal en el rendimiento de fitomasa aérea seca (g/planta) en el cultivo de haba (Toralapa, 1993-94).

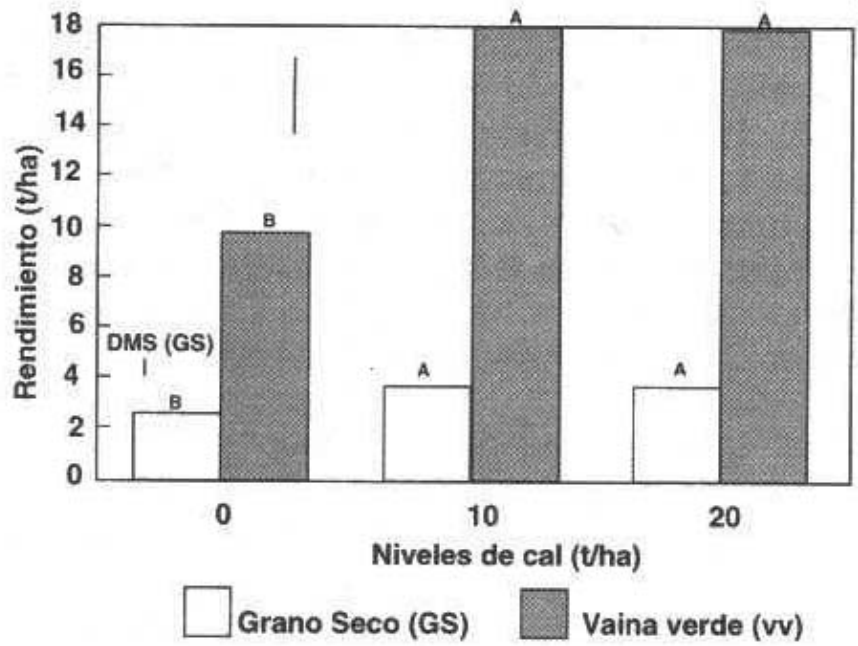

Figura 8. Efecto residual de cal en el rendimiento: en grano seco y en vaina (t/ha), del cultivo de haba (Toralapa, 1993-94). 


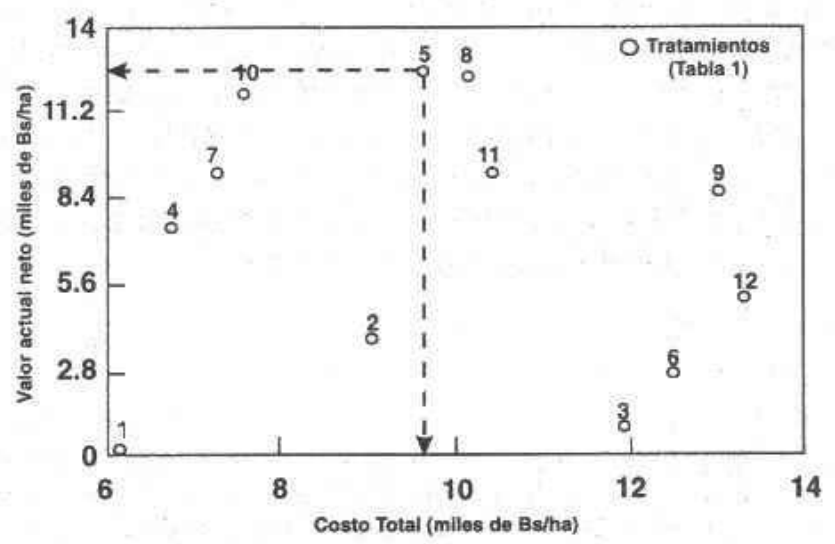

Figura 9. Valor Actual Neto (VAN) de los tratamiento en Bs/ha, en función de los costos totales actualizados (Toralapa, 1991-94).

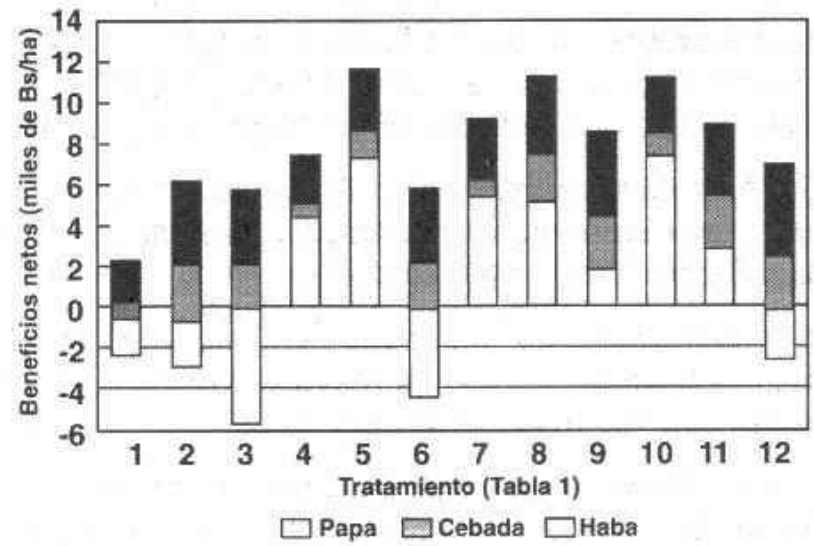

Figura 10. Beneficios netos actualizados, aportados por cada cultivo del sistema de rotación (Toralapa, 1991-94). 
Los resultados presentados muestran que, en condiciones de suelos ácidos, la aplicación de cal combinada con niveles recomendados de fertilizantes tiene un impacto agroeconómico positivo sobre los cultivos manejados por los agricultores en su sistema de producción. Sin embargo, es necesario investigar más esta propuesta, para optimizar su rentabilidad y entender mejor los efectos agronómicos de la aplicación de cal sobre los cultivos de la rotación.

Este trabajo fue ejecutado dentro del marco del convenio del Programa de Investigación de la Papa, PROINPA, que fue establecido entre la Secretaría Nacional de Agricultura y Ganadería de Bolivia, la Cooperación Suiza al Desarrollo (COSUDE) y el Centro Internacional de la Papa (CIP).

\section{Referencias Bibliográficas}

1. Alí, G. C. 1989. Estados de desarrollo y análisis de crecimiento en cuatro variedades de haba (Vicia faba L.) a dos densidades. Tesis Ing. Agrónomo. Facultad de Ciencias Agrícolas y Pecuarias. Cochabamba, Bolivia. 127 p.

2. Centro de Investigación de Agricultura Tropical (CIAT). 1991. Primera Reunión Boliviana de Rhizobiología. CIAT - Academia Nacional de Ciencias de Bolivia - WAU. Santa Cruz, Bolivia, p. 34.

3. _ 1994. Métodos usados en el Proyecto Rhizobiología para estudiar la interacción entre la bacteria rizobiacea y la planta leguminosa. Santa Cruz, Bolivia, p. 3-31.

4. Comité Interinstitucional de Desarrollo de Tiraque (CIDETI). 1994. Diagnóstico socioeconómico de la microregión Tiraque. Volumen 2 Economía. Cochabamba, Bolivia, p. 8-12.

5. 1994. Diagnóstico socioeconómico de la microregión Tiraque. Volumen 7 - Suelos. Cochabamba, Bolivia, p. 8-12.

6. Devaux, A.; Vallejos, J. 1992. Informe Anual (1991-1992). Programa de Investigación de la Papa. Cochabamba, Bolivia, p. 232 - 239.

7. García, A. 1987. Cultivos herbáceos. Ediciones Mundi Prensa. Madrid, España, p. 485. 
8. Herbas, J. 1995. Manejo agronómico del haba de exportación. En: Boletín Técnico Nº 2 - TECNOIBTA. La Paz, Bolivia. p. 2-3.

9. Iriarte, E.; Araníbar, M. 1977. Clasificación taxonómica y capacidad de uso de tierras productoras de papa. Departamento de suelos del M.A.C.A. Cochabamba, Bolivia.

10. Mejía, J.M. 1994. Evaluación de tres factores que afectan la fijación biológica del nitrógeno en haba (Vicia faba L.) en valles y alturas de Cochabamba. Tesis Ing. Agrónomo - UMSS. Cochabamba, Bolivia. $133 \mathrm{p}$.

11. Mokate, K.M.; Cuervo de Forero, A. 1990. La evaluación financiera de proyectos de inversión. Centro de estudios sobre desarrollo económico - Universidad de los Andes. Bogotá, Colombia, p. 66-117.

12. Pandey, R.K.; Herrer, W.A.T.; Pendleton, J.W. 1984. Yield and yield components. En: Drought response of grain legumes under irrigation gradient. Agronomy, Journal. Wisconsin, EE.UU. p. 549 -552.

13. Pijnenborg, J. 1993. la peletización de semillas como práctica para mejorar la fijación biológica del nitrógeno. En: $1^{\circ}$ Reunión Nacional de Leguminosas de grano y $2^{\circ}$ reunión Boliviana de Rhizobiología. Cochabamba, Bolivia. $64 \mathrm{p}$.

14. Quiroga, J.; Blajos, J. 1995. Revisión de métodos para el análisis económico en papa. Departamento de Ciencias Sociales -Programa de Investigación de la Papa. Cochabamba, Bolivia. Doc. 3/95.

15. Roberts, M.J., Long, S.P., Tieszen, L.L.; Beadle, C.L. 1985. Medición de la biomasa vegetal y de la producción primaria neta En: Técnicas en fotosíntesis y bioproductividad. Programa Ambiental de las Naciones Unidas. Chapingo, México, p. 3-8.

16. Sánchez, P. 1981. Suelos de trópico. Características y manejo. Trad. Camacho, E. MCA. San José, Costa Rica. 625 p.

17. Villarroel, J. 1988. Manual práctico para la interpretación de análisis de suelos en Cochabamba. Edición AGRUCO, F.C.A.P.-U.M.S.S. Cochabamba, Bolivia. $33 \mathrm{p}$.

18. Willard, H.G. 1992. Manual de fertilizantes. National Plant Food Institute. Ediciones Limusa. México D.F. 285 p. 